http://dx.doi.org/10.35381/s.v.v5i9.1110

\title{
Valoración nutricional y características sociodemográficas de adolescentes escolarizados
}

Nutritional assessment and sociodemographic characteristics of school adolescents

\author{
Doménica Vanesa Posso-López \\ dvpossol@utn.edu.ec \\ Universidad Técnica del Norte, Ibarra \\ Ecuador \\ https://orcid.org/0000-0002-6221-1853 \\ Giovanna Paulina Posso-López \\ gposso9410@uta.edu.ec \\ Universidad Técnica de Ambato, Ambato \\ Ecuador \\ https://orcid.org/0000-0003-1639-1715
}

Recepción: 01 de octubre 2020

Revisado: 01 de noviembre 2020

Aprobación: 20 de diciembre 2020

Publicación: 01 de enero 2020 


\title{
RESUMEN
}

Objetivo: Realizar la evaluación del estado nutricional y sus características sociodemográficas de los adolescentes de la Unidad Educativa Alberto Enríquez, Imbabura, Atuntaqui, Ecuador Método: Se utilizó el enfoque cuantitativo de tipo descriptivo y corte transversal, con una población en estudio de 66 adolescentes. Resultados: Predominio en el sexo femenino con un $60.6 \%$ frente al sexo masculino con el $39,4 \%$, con un predominio en la etnia mestiza con el $71,2 \%$. Con respecto a la evaluación nutricional dietética se evidenció que existe un gran consumo de sal, grasas, cereales y frutas, con un bajo consumo de hortalizas y tubérculos. Conclusión: El consumo elevado de grasas y sal puede afectar el normal desarrollo y crecimiento de los adolescentes, el mismo que predispone al desarrollo de enfermedades crónicas no transmisibles.

Descriptores: Medicina del adolescente; fenómenos fisiológicos de la nutrición; endocrinología; agentes comunitarios de salud. (Fuente: DeCS, 2020).

\begin{abstract}
Objective: To carry out the evaluation of the nutritional status and its sociodemographic characteristics of the adolescents of the Alberto Enríquez Educational Unit, Imbabura, Atuntaqui, Ecuador. Method: The quantitative, descriptive and cross-sectional approach was used, with a study population of 66 adolescents. Results: Prevalence in the female sex with $60.6 \%$ compared to the male sex with $39.4 \%$, with a predominance in the mestizo ethnic group with $71.2 \%$. Regarding the dietary nutritional evaluation, it was evidenced that there is a large consumption of salt, fat, cereals and fruits, with a low consumption of vegetables and tubers. Conclusion: The high consumption of fat and salt can affect the normal development and growth of adolescents, the same that predisposes to the development of chronic noncommunicable diseases.
\end{abstract}

Descriptors: Adolescent medicine; nutritional physiological phenomena; endocrinology; community health workers. (Source: DeCS, 2020). 


\section{INTRODUCCIÓN}

La adolescencia es un periodo crítico del desarrollo y crecimiento del ser humano, durante el mismo se produce una serie de cambios físicos, fisiológicos, biológicos, psicológicos y sociales. Se establecen y definen ciertas características del adulto, por tal motivo es importante realizar una correcta evaluación nutricional del individuo (1). La evaluación nutricional en este grupo poblacional es imprescindible ya que son indicadores de crecimiento para poder clasificar su estado nutricional e identificar de manera precoz factores de riesgo que pueden afectar su correcto desarrollo y crecimiento. Las ingestas dietéticas para los adolescentes son creadas en base de su edad y sexo (2),(3).

Los adolescentes tienen necesidades nutricionales elevadas debido a su gran velocidad de crecimiento que es el pico máximo durante el ciclo de vida, dónde adquieren el $25 \%$ de la talla definitiva y el $50 \%$ del peso final. En esta etapa crítica de la adolescencia existe un factor de riesgo para la salud, son los hábitos perniciosos ${ }^{(4)}$. La adolescencia es un ciclo de vida donde se evidencian cambios sociales y psicológicos que pueden verse afectados, es un momento de la vida donde se experimentan diversas actividades que son adictivas y peligrosas para la salud(5).

El consumo de alcohol conlleva a una serie de complicaciones que suelen ser crónica ${ }^{(6)}$. El continente de América ocupa el segundo lugar entre las regiones de la Organización Mundial de la Salud con los niveles más altos en consumo excesivo de alcohol, el promedio de consumo se da a partir de los 15 años en adelante ${ }^{(7)}$.

El consumo de tabaco es un factor de riesgo común de las enfermedades cardiovasculares, cáncer, enfermedades respiratorias. El consumo de tabaco es una gran amenaza que enfrenta la salud a nivel mundial. La mayor parte de personas iniciaron el hábito tabáquico empezaron en la adolescencia, un grupo poblacional vulnerable con gran facilidad de crear dependencia (8) (9).

La Organización Mundial de la Salud (OMS) refiere que existe otro problema de salud pública un a alimentación poca saludable, siendo una problemática social y sanitaria a nivel mundial. La OMS reconoce que existe la mal nutrición, en donde se incluye la subnutrición y las deficiencias nutricionales, siendo una de las principales causas de 
morbi-mortalidad a nivel mundial ${ }^{(10)}$. El $79 \%$ de las causas de mortalidad se encuentran atribuidas a las enfermedades crónicas no transmisibles, sobre todo en los países en vías de desarrollo, siendo las más comunes afecciones cardio vasculares, diabetes mellitus, obesidad, síndrome metabólico, las mismas que pueden ser tratadas de manera eficaz y con intervenciones económicas, en particular las acciones de promoción y prevención de salud, relacionadas con el régimen alimentario, actividad física, intervenciones sobre los hábitos perniciosos y el modo de estilo(11).

\section{Objetivo General}

Evaluar el estado nutricional, conocer sus características sociodemográficas y hábitos perniciosos en los adolescentes de la Unidad Educativa "Alberto Enríquez" Atuntaqui, Imbabura, Ecuador.

\section{MÉTODO}

\section{Tipo de investigación}

Es un estudio de tipo descriptivo que cuantifica y analiza las diferentes variables, La muestra estará conformada por 60 adolescentes estudiantes de la institución, entre edades de 15 y 18 años.

Para la recolección de datos sociodemográficos se utilizó un cuestionario que constató la edad, sexo, lugar de procedencia y nivel de escolaridad.

La valoración de la evaluación nutricional se realizó a través del patrón de consumo de alimentos y se agruparon alimentos como verduras, frutas, azúcar, grasa y sal, tomando en cuenta la siguiente escala:

1. Al día (Uno, dos a tres veces)

2. Semanal (uno, dos a tres, mayor o igual a cuatro)

3. Mensual (uno a tres veces)

4. Nunca

Para conocer los hábitos perniciosos se tomó en cuenta dos hábitos: Consumo de alcohol y tabaco. 
1. Para identificar el consumo de alcohol y la frecuencia del mismo de los adolescentes se utilizó una encuesta de opción simple, donde el primer aspecto fue definir si existe o no consumo de alcohol, si la respuesta es positiva se indica a seguir con la siguiente pregunta, donde indica la frecuencia del mismo.

2. Para conocer el consumo de cigarro del estudiante se evaluó por medio de una pregunta cerrada y $s$ frecuencia si la respuesta fuera positiva.

3.

Tabla 1. Caracterización sociodemográfica de los estudiantes de la Unidad educativa "Alberto Enríquez", Atuntaqui, Imbabura.

\begin{tabular}{|l|l|l|}
\hline Características sociodemográficas & $\%$ \\
\hline Edad & 15 & 3 \\
& 16 & 33,3 \\
& 17 & 51,5 \\
& 18 & 12,1 \\
& Total & 100 \\
\hline \multirow{2}{*}{ Sexo } & Hombre & 39,4 \\
& Mujer & 60,6 \\
& Total & 100 \\
\hline Etnia & Blanco & - \\
& Afrodescendiente & - \\
& Mestizo & 71,2 \\
& Indígena & 28,8 \\
& Total & 100 \\
\hline Lugar de procedencia & Urbano & 48,5 \\
& Rural & 51,5 \\
& Total & 100 \\
& & \\
\hline
\end{tabular}

Fuente: Cuestionario aplicado. 
Tabla 2. Identificación de los hábitos perniciosos en los adolescentes de la Unidad Educativa "Alberto Enríquez", Atuntaqui, Imbabura.

\begin{tabular}{|l|l|l|}
\hline \multicolumn{3}{|c|}{ Hábitos perniciosos } \\
\hline Consumo de bebidas alcohólicas & si & $22,7 \%$ \\
& no & $77,3 \%$ \\
\hline Consumo de cigarrillo & si & $6,1 \%$ \\
& no & $93,9 \%$ \\
\hline
\end{tabular}

Fuente: Cuestionario aplicado.

Tabla 3. Identificación de la frecuencia de consumo de alimentos.

\begin{tabular}{|l|c|c|c|c|c|}
\hline & \multicolumn{5}{|c|}{ Frecuencia de consumo } \\
\hline \multicolumn{1}{|c|}{ Alimento } & $\begin{array}{c}\text { 1 vez } \\
\text { al dia } \\
\%\end{array}$ & $\begin{array}{c}2-4 \text { veces } \\
\text { por } \\
\text { semana } \%\end{array}$ & $\begin{array}{c}\text { 1 vez a la } \\
\text { semana \% }\end{array}$ & $\begin{array}{c}\text { 2 veces } \\
\text { por mes } \\
\%\end{array}$ & $\begin{array}{c}\text { Nunca o } \\
\text { casi } \\
\text { nunca \% }\end{array}$ \\
\hline $\begin{array}{l}\text { Leche } \\
\text { derivados }\end{array}$ & 4,54 & 22,22 & 35,35 & 23,73 & 14,14 \\
\hline Huevo & 25,8 & 43,9 & 18,2 & 6,1 & 6,1 \\
\hline Carnes & 1,76 & 22,97 & 29,54 & 28,28 & 17,42 \\
\hline Leguminosas & 0,30 & 24,24 & 42,42 & 20 & 13,03 \\
\hline Cereales & 15,36 & 28,35 & 22,29 & 16,88 & 15,36 \\
\hline Tubérculos & 9,84 & 15,90 & 10,60 & 19,69 & 43,93 \\
\hline Hortalizas & 4,92 & 25,94 & 18,56 & 18,93 & 31,62 \\
\hline Frutas & 9,46 & 26,89 & 25,88 & 19,06 & 18,68 \\
\hline Azúcares & 16,16 & 20,20 & 16,66 & 17,67 & 29,29 \\
\hline Grasas & 7,07 & 28,78 & 22,72 & 12,62 & 27,77 \\
\hline Sal & 47 & 45,5 & 3 & 1,5 & 3 \\
\hline Comidas & 2,27 & 6,06 & 14,01 & 20,83 & 56,81 \\
rápidas & & & & & \\
\hline
\end{tabular}

Fuente: Cuestionario aplicado. 
Tabla 4. Identificación de Enfermedades Crónicas no Transmisibles, como antecedentes patológicos familiares de los estudiantes de la Unidad Educativa "Alberto Enríquez, Atuntaqui, Imbabura.

\begin{tabular}{|l|l|}
\hline Antecedentes patológicos & $\%$ \\
\hline Sobrepeso u obesidad & 10,6 \\
Diabetes & $\mathbf{2 8 , 8}$ \\
Hipertensión & $\mathbf{2 4 , 2}$ \\
Derrame cerebral & 6,1 \\
Enfermedad del corazón & 15,2 \\
Colesterol o triglicéridos elevados & 10,6 \\
Cáncer & 16,7 \\
Enfermedades respiratorias crónicas & 4,5 \\
Ninguno & $\mathbf{4 7}$ \\
\hline
\end{tabular}

Fuente: Cuestionario aplicado.

A continuación, se presentan en la figura 1 la representación gráfica de los resultados obtenidos de la investigación de campo
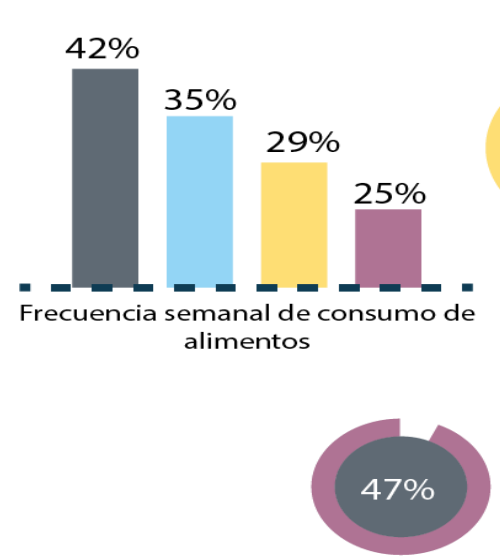

NINGUNA

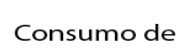

$$
\text { cigarrillo }
$$

$6.1 \%$
Consumo de bebidas alcohólicas
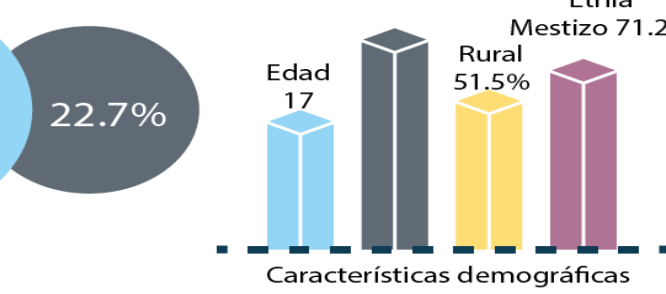

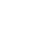




\section{DISCUSIÓN}

En la tabla 1, se observa que la edad que más predomina en los adolescentes de la Unidad Educativa que participaron fue de 17 años con el 51,5\%, la mayor parte de población corresponde al sexo femenino con el $60,6 \%$ del total, además, la etnia que sobresale en este estudio fue la mestiza con un $71,2 \%$ y la indígena con $28,8 \%$, respecto al lugar de procedencia se obtuvo que el 52,5\% pertenecen al sector rural. Los datos obtenidos de hombres y mujeres guardan relación con los registros del INEC 2016 que presentan $50,5 \%$ mujeres y el $49,4 \%$ hombres $^{(12)}$.

En la tabla 2, sobre los hábitos perniciosos se evidencia que existe bajo consumo de alcohol en los adolescentes, el 77,3\% no consumen bebidas alcohólicas; y los que si lo hacen tienen una frecuencia de 1 a 3 veces por año.

Estas cifras son bajas en comparación con las presentadas en ENSANUT 2012, donde el consumo de alcohol en adolescentes de 15 a 18 años es de $71,7 \%{ }^{(13)}$.

En un estudio realizado en Brasil en el año 2020 sobre "Asociación entre estilo de vida comportamientos y calidad de vida relacionada con la salud en una muestra de adolescentes brasileños" se evidencia que el consumo de bebidas alcohólicas es del $43,1 \%$. En relación a los datos obtenidos del presente estudio son bajos 22,7\% (14).

En cuanto al segundo hábito pernicioso el 93,6 \% de adolescentes no consumen cigarrillo, sin embargo, los que si fuman (6,1\%) lo hace una a tres veces al año y consume hasta cinco unidades.

En cuanto al consumo de cigarrillos estas cifras son menores a las presentadas en el ENSANUT 2012, en donde el consumo de cigarrillo en adolescentes fue de $26,4 \%$.

En un estudio realizado en Brasil en el año 2020 sobre "Evaluación de la obesidad asociada a factores de riesgo para la salud en la escuela pública brasileña" se evidencia que el $94,4 \%$ de adolescentes no fuman. En cuanto a los datos del estudio realizado son bajos el $93,9 \%{ }^{(15)}$.

En la tabla 3, Identificación de la frecuencia de consumo de alimentos en los estudiantes de la Unidad Educativa "Alberto Enríquez", Atuntaqui, Imbabura; se demostró que existe un consumo alto de cereales, grasas, huevos, frutas y sal; consumo moderado de lácteos, carnes y leguminosas; y bajo consumo de tubérculos, 
hortalizas, azucares y comidas rápidas. Los datos relevantes y de riesgo nutricional son consumo alto de grasas $28,78 \%$ (dos a cuatro veces por semana), sal $47 \%$ ( 1 vez al día) y un bajo consumo de hortalizas $31,62 \%$.

En un estudio realizado en Paraguay en el año 2020 sobre "Caracterización Alimentaria nutricional de adolescentes de la comunidad campesina del Barrio Ybyraty de Paraguarí" se evidencia que el consumo de grasas y aceites es el más consumido a diario con el 100\% (16). En relación con el promedio obtenido de consumo de grasas en la Unidad Educativa "Alberto Enríquez" el consumo de grasas es bajo $(7,07 \%$ al día).

En un estudio realizado en Cuba, en el año 2015 referente a "Estilos de vida, hipertensión arterial y obesidad en adolescentes" el 23,5\% agrega sal a los alimentos después de cocinarlos ${ }^{(16)}$.

Según en el estudio realizado en Cuba, en el año 2015 referente a "Estilos de vida, hipertensión arterial y obesidad en adolescentes" se observa el $36,6 \%$ consume vegetales 2 a 4 veces a la semana y 12,3\% todos los días (17). Estos datos son mayores al presente estudio porque existe un consumo promedio de verduras de $28,35 \%$ entre 2 a 4 veces a la semana.

En la tabla 4, sobre la identificación de factores de riesgo como son las Enfermedades crónicas no transmisibles como antecedentes patológicos familiares de los adolescentes, los más relevantes son diabetes el 28,8\%, hipertensión $24,2 \%$ y cáncer $16,7 \%$; y quienes no presentan antecedentes patológicos familiares son el $47 \%$.

En un estudio realizado en Honduras en el año 2015 sobre "Prevalencia de sobrepeso/obesidad, evolución antropométrica y factores relacionados en adolescentes de institutos de educación media, Tegucigalpa, Honduras" evidencia que no presentan familiares con ECNT el $63,63 \%{ }^{(18)}$. En relación con el estudio realizo en la Unidad Educativa estos datos son más elevados.

\section{CONFLICTO DE INTERÉS}

Las autoras declaran que no tienen conflicto de interés en la publicación de este artículo. 


\section{FINANCIAMIENTO}

Autofinanciado.

\section{AGRADECIMIENTO}

A todas las autoridades y personal de la Unidad Educativa Alberto Enríquez; por permitir la realización de todo el proceso investigativo dentro del establecimiento educativo.

Asimismo, agradecemos a la Universidad Técnica del Norte y a toda la Carrera de Nutrición y Salud Comunitaria.

\section{REFERENCIAS}

1. Gaete V. Adolescent psychosocial development. Rev Chil Pediatr. 2015;86(6):436-43.

2. Ministerio de salud. Valoración Nutricional|Alimentación Saludable [Nutritional Assessment | Healthy Eating] [Internet]. [cited 2020 Aug 18]. Available from https://n9.cl/713oa

3. Valderrama S., López M. PG. Valoración del estado nutricional y hábitos alimentarios en niños de 1 a 3 años de edad que acuden a la guardería "Bahía" de la ciudad de Guayaquil en el periodo 2018-2019. 2019;13-5 [Assessment of the nutritional status and eating habits in children between 1 and 3 years of age who attend the "Bahía" nursery in the city of Guayaquil in the period 2018-2019. $2019 ; 13-5]$. Available from https://n9.cl/x0ws8

4. Organización Mundial de la Salud. Adolescentes: riesgos para la salud y soluciones [Adolescents: health risks and solutions] [Internet]. [cited 2020 Aug 18]. Available from https://n9.cl/ebbm

5. Organización Mundial de la Salud. Desarrollo en la adolescencia [Development in adolescence]. [Internet]. 2015 [cited 2020 Aug 18]; Available from https://n9.cl/57eq

6. Góngora Gómez O, Elizabeth Gómez Vázquez Y, Antonio M, Ojeda R, Leandro $\mathrm{J}$, Guerrero $\mathrm{P}$, et al. Comportamiento del alcoholismo en adolescentes [Behavior of alcoholism in adolescents]. Acta Médica del Cent. 2019;13(3):31527. 
7. OPS. Prevención Integral de Enfermedades Crónicas no Transmisibles [Comprehensive Prevention of Chronic Noncommunicable Diseases] [Internet]. 2014 [cited 2020 Aug 18]. Available from https://n9.cl/ngg90

8. Gualayin Pablo, Irazola Vilma, Rubinstein Adolfo, Bruno Rodrigo, Días André, Gulayin Miguel UM y BA. Adolescentes Libres de Tabaco. Efectividad de una intervención educativa [Tobacco Free Teens. Effectiveness of an educational intervention]. 2018;116(3):392-400.

9. Metro Ecuador ¿A qué edad los jóvenes comienzan a consumir alcohol? [At what age do young people begin to consume alcohol?] [Internet]. 2017 [cited 2020 Aug 18]. Available from https://n9.cl/w5zma

10. Organización Mundial de la Salud. Alimentación sana [Healthy food] [Internet]. 2018 [cited 2020 Aug 18]. Available from https://n9.cl/ihqu

11. Rosales Ricardo Y, Peralta L, Yaulema L, Pallo J, Orozco D, Caiza V, et al. Alimentación saludable en docentes [Healthy eating in teachers]. Rev Cuba Med Gen Integr. 2017;33(1):115-28.

12. Instituto nacional de estadistica y censos 2016 [Internet]. [cited 2020 Oct 11]. Available from https://n9.cl/v6r9e

13. Encuesta Nacional de Salud y Nutrición ENSANUT-ECU 2012 [Internet]. 2014 [cited 2020 Aug 18]. Available from https://n9.cl/se8f

14. Da Costa BGG, Chaput JP, Lopes MVV, da Costa RM, Malheiros LEA, Silva KS. Association between lifestyle behaviors and health-related quality of life in a sample of Brazilian adolescents. Int $J$ Environ Res Public Health. 2020;17(19):1-11.

15. Corazzini R, Morgado F, Gascón TM, Affonso Fonseca FL. Evaluation of obesity associated with health risk factors in Brazilian public school. Obes Med. 2020;19(January).

16. Costas-Duarte V, Alfonzo-Maciel F, Serafini-Melgarejo M, Flores-Alatorre JF. Caracterización Alimentaria nutricional de adolescentes de la comunidad campesina del Barrio Ybyraty de Paraguarí [Nutritional food characterization of adolescents from the rural community of the Ybyraty neighborhood of Paraguarí]. Memorias del Inst Investig en Ciencias la Salud. 2020;18(1):22-31.

17. González Sánchez R, Llapur Milián R, Díaz Cuesta M, Illa Cos MDR, Yee López $\mathrm{E}$, Pérez Bello D. Estilos de vida, hipertensión arterial y obesidad en adolescentes [Lifestyles, high blood pressure and obesity in adolescents]. Rev Cubana Pediatr. 2015;87(3):273-84. 
18. Rodríguez K, Rodas P, Mairena D, Sánchez C. Prevalencia De Sobrepeso/ Obesidad, En Adolescentes De Institutos De Educación Media [Prevalence of Overweight / Obesity in Adolescents from Secondary Education Institutes]. 2015;83:7-9.

2021 por los autores. Este artículo es de acceso abierto y distribuido según los términos y condiciones de la licencia Creative Commons Atribución-NoComercial-Compartirlgual 4.0 Internacional (CC BY-NC-SA 4.0) (https://creativecommons.org/licenses/by-nc-sa/4.0/). 